Décadrages Décadrages

cınéma, à travers champs Cinéma, à travers champs

$43 \mid 2020$

Abdellatif Kechiche

\title{
Le Milieu de l'horizon, de Roland Buti à Delphine Lehericey : l'adaptation au prisme du genre
}

Alain Boillat

\section{(2) OpenEdition}

1 Journals

Édition électronique

URL : https://journals.openedition.org/decadrages/1595

DOI : 10.4000/decadrages. 1595

ISSN : 2297-5977

Éditeur

Association Décadrages

Édition imprimée

Date de publication : 1 juillet 2020

Pagination : 136-147

ISSN : 2235-7823

\section{Référence électronique}

Alain Boillat, «Le Milieu de I'horizon, de Roland Buti à Delphine Lehericey : I'adaptation au prisme du genre », Décadrages [En ligne], 43 | 2020, mis en ligne le 15 octobre 2021, consulté le 06 mars 2022. URL : http://journals.openedition.org/decadrages/1595; DOI : https://doi.org/10.4000/decadrages. 1595

(B) Décadrages 


\section{Roland Buti \\ Le Milieu \\ de l'horizon}

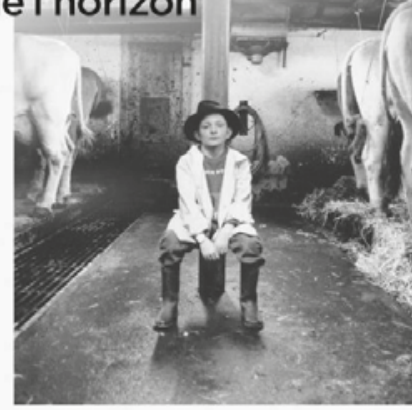

ZOE

Poche

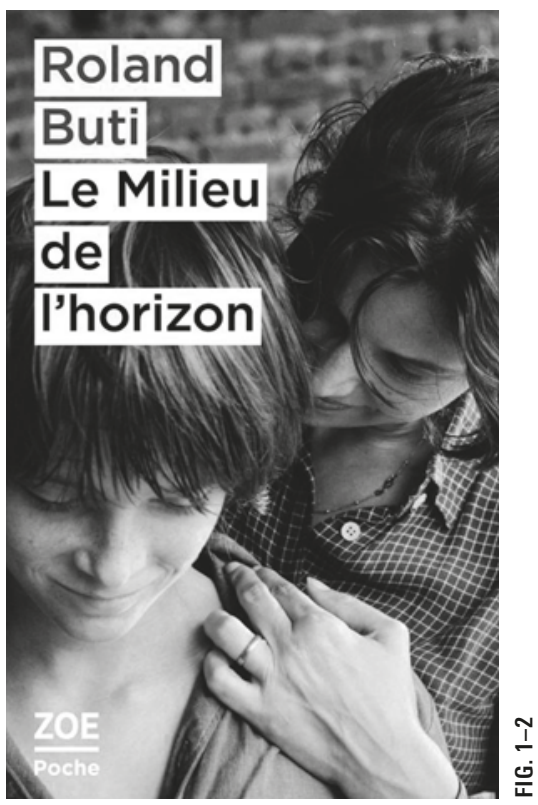

Pour l'image de couverture de la première édition du Milieu de l'horizon en 2013, Zoé optait pour une photographie documentaire en noir et blanc de Vanessa Püntener, portrait frontal d'un garçon saisi dans un environnement agricole dont la présence s'affirme grâce à une forte profondeur de champ. La réédition du roman en 2019 remplace cette iconographie parente de certaines images du cinéma de Fredi M. Murer par une illustration en couleur tirée du film de Lehericey qui plonge d'entrée de jeu le lecteur dans l'intimité des rapports mère-fils que l'adaptation cinématographique met en exergue; la coïncidence des bords de l'image en plan rapproché avec la page suggère le prélèvement de cet instant sur un univers (filmique) qui l'excède. 
LE MILIEU DE L'HORIZON,

DE ROLAND BUTI À DELPHINE LEHERICEY: L'ADAPTATION AU PRISME DU GENRE
Bien que réalisé par la cinéaste belge Delphine Lehericey, le film Le Milieu de l'horizon (CH/B, 2019), coproduit par la maison de production lausannoise Box Productions (à l'instar de la précédente fiction de la réalisatrice, Puppylove, en 2013), affirme sa "suissitude» en tant qu'adaptation de l'œuvre homonyme écrite par le Suisse romand Roland Buti - parue aux éditions Zoé en $2013^{(\text {Fig.1-2) }}$, elle a connu à sa sortie une reconnaissance certaine, notamment en décrochant le Prix suisse de littérature l'année suivante ${ }^{1}$-, et dont l'action se déroule en terres vaudoises au cours de la grande sécheresse qui sévit en 1976. Certes, aucune date ni indication géographique ne figure au début du film, tandis qu'aux seuils du roman se trouvent en aval une épigraphe en l'espèce d'une citation d'un texte documentaire signé par le représentant de la Division fédérale de l'agriculture paru dans La Gazette de Lausanne du 26 juin 1976, et en amont une quatrième de couverture qui précise l'année durant laquelle se déroule la quasi-intégralité de I'histoire (seul un épilogue a lieu plusieurs années plus tard qui n'a pas été retenu dans le film).

D'ailleurs, les répliques du Milieu de l'horizon, dépourvues de couleur locale, comprennent des expressions d'aujourd'hui (la réitération strictement phatique de la locution "du coup" se démarque par exemple comme un tic langagier actuel). Même si les voitures présentes à l'écran, dont on peut noter que les plaques d'immatriculation ne sont pas suisses, contribuent à situer l'intrigue dans les années $1970^{2}$, ce souci de "reconstitution» ne paraît pas prioritaire. Le film a en effet été tourné en Macédoine, ce qui l'inscrit dans une tradition différente de celle de l'ancrage local d'un film comme Les Petites fugues $(\mathrm{CH} / \mathrm{F}, 1979)^{3}$, lequel présente néanmoins plusieurs parentés avec Le Milieu de l'horizon (il s'agit aussi d'un récit initiatique ou d'émancipation en milieu paysan dans lequel l'évolution d'un personnage s'exprime par des déplacements sur un deux roues, et où le développement des individus s'opère sur fond de mutations de l'agriculture traditionnelle) ${ }^{4}$. En ne privilégiant pas l'amarrage de la fiction à un substrat documentaire qui résiderait en premier lieu dans un espace géographique identifiable, le film de Lehericey exploite
De son côté, le film a été nominé pour le Prix du cinéma suisse 2020 dans les catégories "meilleur film de fiction" (Delphine Lehericey), "meil-

leur scénario » (Joanne Giger), "meilleure interprétation masculine" (Luc Bruchez) et "meilleure musique de film» (Nicolas Rabaeus) - il y a obtenu les Quartz du meilleur film et du meilleur scénario.

2 II n'en demeure pas moins que la Renault 5 du personnage de Cécile, dont l'intrusion dans l'espace de la ferme est aux prémices de bouleversements radicaux dans la famille des Sutter, est en fait une Renault Super 5 commercialisée à partir de l'automne 1984. La vraisemblance, on le sait, dépend de "l'encyclopédie" du spectateur, et nous ne ferons pas grief à la production du film d'avoir postulé un public plus passionné de cinéma (tournage en $35 \mathrm{~mm}$ ) que d'automobiles.

$3 \quad$ Notons toutefois que pour Les Petites fugues, où l'époque de I'histoire racontée est celle de la sortie du film, Yves Yersin ne s'est pas contenté de recourir à des interprètes helvétiques: le personnage principal de Pipe est en effet incarné par Michel Robin, qui fut sociétaire de la Comédie française.

4 Le film de Yersin est, comme Le Milieu de I'horizon, ponctué de scènes de repas filmées dans des intérieurs qui ont été reconstitués pour le tournage (en Suisse alémanique pour le premier, en Belgique pour le second) au cours desquelles éclatent des conflits entre les générations. Les personnages discutent surtout de la nécessité d'abandonner un certain modèle de gestion de l'exploitation au profit d'un autre: le fils qui devrait reprendre la ferme prend contact dans Les Petites fugues avec des conseillers qui recommandent d'abandonner l'agriculture au profit de l'élevage, ce qui implique un changement analogue à celui provoqué par la mise à exécution du projet de poussinière dans Le Milieu de l'horizon (dont le récit est toutefois centré sur l'activité professionnelle du père). 
5 Puppylove (B/CH/F/Lux, 2013) est I'histoire d'une adolescente qui s'éveille à la sexualité et entretient un rapport fusionnel avec son père, tout comme Gus avec sa mère dans Le Milieu de I'horizon; sa vie intime est bouleversée par l'arrivée d'une voisine de son âge qui l'entraîne dans une série d'expériences, qui s'amuse à la séduire et I'éloigne momentanément de son père. Les thématiques de ce film (initiation à la sexualité, éclatement de la cellule familiale qui est monoparentale dans Puppylove, homosexualité féminine) sont également déclinées dans le roman de Buti.

6 La description de l'expérience de lecture de ces récits à suivre destinés à la jeunesse est particulièrement fine sous la plume de Buti (voir en particulier Roland Buti, Le Milieu de I'horizon, Carouge-Genève, Zoé, 2017, pp. 12-13, 66-67, et 115) même si, d'un point de vue historique, elle correspond mieux à une génération précédente du lectorat (la jeunesse francophone de l'aprèsguerre et des années 1950). À propos de ce régime de lecture, nous nous permettons de renvoyer à Alain Boillat, Françoise Revaz, Marine Borel et Raphaël Oesterlé, Case, strip, action! Les feuilletons en bandes dessinées dans les magazines pour la jeunesse (1946-1959), Gollion, Infolio, 2016.

7 Par exemple dans des formules telles que celle-ci: "Cette réponse, et l'idée de la possibilité d'une distribution des chromosomes aléatoires à la naissance, m'a troublé pendant des années" (R. Buti, op. cit., p. 15).

$8 \quad$ Id., pp. 9-10. plutôt une veine allégorique, se présentant à cet égard comme la réitération d'une formule précédemment utilisée (avec succès) par Box Productions pour Home d'Ursula Meier (CH/F/B, 2008), un film également consacré à une forme d'éclatement d'une cellule familiale située en marge de la société à travers l'évolution de protagonistes appartenant à des générations différentes et confrontés à un bouleversement qui affecte leur environnement quotidien. À la réalisatrice qui ne connaissait pas l'ouvrage de Buti (voir l'entretien infra), la maison de production a offert clé en main un projet de "film d'auteur" qui instaure par ailleurs une forme de continuité avec son long-métrage précédent ${ }^{5}$. Comme Home, Le Milieu de I'horizon résulte d'une co-production entre la Suisse et la Belgique (pays auxquels s'ajoute la France pour le premier) et proposant un casting mêlant une vedette française pour incarner la mère (Isabelle Huppert / Laetitia Casta) et un jeune Suisse inconnu du public - ce qui garantit la médiatisation de la sortie via la presse locale - dans le rôle du fils (Kacey Mottet Klein / Luc Bruchez). Cependant, alors que Home est quant à lui basé sur un scénario original (ré)écrit à plusieurs mains, Le Milieu de l'horizon assume et même revendique son statut d'adaptation (ainsi qu'on l'observe dans le matériel promotionnel).

\section{LE POINT DE VUE MASCULIN DU ROMAN}

Dans Le Milieu de I'horizon de Buti, le lecteur est invité à partager le quotidien et les pensées d'un jeune fils de paysans, Gus, durant un été caniculaire qu'il passe à la ferme de ses parents avec son cousin Rudy atteint de retard mental, jusqu'au drame que constitue le décès de Rudy - dans le film, il tombe dans le coma mais reste en vie - qui, lorsqu'éclate une averse qui met violemment fin à la sécheresse, se noie dans la poussinière avec laquelle le père a désespérément tenté de réorienter ses activités pour éviter la faillite, impuissant face aux ravages de la chaleur excessive à laquelle succombent ses poussins, puis à la destruction provoquée par la tempête. Dès l'ouverture de ce roman écrit à la première personne qui à la fois nous plonge dans l'imaginaire de l'enfant nourri des récits d'aventures qu'il lit dans des périodiques de bandes dessinées ${ }^{6}$ comme autant d'échappées hors d'une vie rurale monotone et maintient en filigrane une certaine distance en soulignant le caractère rétrospectif de la narration (il s'agit d'un regard d'adulte porté sur son passé) ${ }^{7}$, nous partageons le regard de l'enfant sur le monde qui l'entoure: "C'était le début des grandes vacances de mes treize ans. [...] Je pensais que cette chaleur particulière était causée par un astéroïde tombé non loin de chez nous, par un gros corps céleste constitué d'un métal inconnu dégageant des vapeurs toxiques invisibles ${ }^{8}$. La cohérence du récit procède de cette focalisation interne stricte, qui a pour effet que les autres personnages, "maman", "papa» (dont on connaît le prénom: Jean), Rudy, la sœur Léa ou Cécile qui vient bouleverser la vie de la famille, ne sont présentés qu'au travers du filtre de la subjectivité de Gus dont le roman retrace l'éveil à la sexualité - le film ménage à cet égard moins 
de progression ${ }^{9}$, sans doute pour mieux correspondre à l'expérience des adolescents d'aujourd'hui -, d'un rapport œdipien à la mère ${ }^{10}$ à une première expérience sexuelle avec sa copine Mado (plus allusive dans le film) en passant par l'attirance pour Cécile. La description du quotidien s'accompagne dans le roman d'éléments ostensiblement porteurs d'une dimension symbolique que l'on pourrait envisager comme des connotateurs de "littérarité". Ainsi Gus recueille-t-il une colombe blessée avec laquelle il entretient une relation qui se développe en miroir de ses sentiments et de sa propre évolution vers I'âge adulte. Sans doute peut-on se réjouir qu'un tel élément, d'abord retenu dans le scénario ${ }^{11}$, ne soit en fin de compte pas venu alourdir le récit du film. II n'en demeure pas moins que le choix initial de le maintenir témoigne de la manière scrupuleuse dont la scénariste Joanne Giger a voulu respecter le roman: le film n'est pas seulement vaguement inspiré du roman de Buti, mais, sans doute pour capitaliser sur le succès de l'œuvre, il se présente comme une adaptation «fidèle " de celle-ci. C'est en raison de cette démarche qu'il est pertinent d'examiner certaines différences entre le film et le roman, au-delà des nécessaires implications du transfert sémiotique d'un moyen d'expression à l'autre et des contraintes spécifiques au contexte de production d'un film. En recourant ponctuellement à l'étude d'états et variantes scénaristiques du Milieu de I'horizon - un traitement de décembre 2014 (soit peu après la parution du roman), une continuité du 3 décembre 2015 ainsi que trois versions ultérieures datées de 2018 - qui, explicitant certaines intentions auctoriales par le biais du langage verbal, ajoutent une strate aux objets soumis à la comparaison, nous examinerons ce film écrit et réalisé par deux femmes dans une perspective d'étude sur le genre ${ }^{12}$, qui nous paraît particulièrement adéquate ici pour rendre compte de la réappropriation personnelle, dans une optique presque féministe, de la matière narrative dont témoigne le film. À cet égard, l'une des facettes du récit, exacerbée dans le film où "maman » sort de l'anonymat (et de la soumission au seul rôle maternel) en recevant le prénom de Nicole (ne serait-ce que parce qu'elle est interprétée par la vedette Laetitia Casta), nous importe tout particulièrement: il s'agit du portrait de la mère, de sa relation aux autres membres de la famille et de son amour pour la plus jeune Cécile, passion qui la conduira à faire le choix de quitter ses proches pour vivre pleinement cette relation adultère. II s'agit surtout du regard posé dans le film sur cette relation homosexuelle et plus généralement sur les personnages féminins, qui à notre sens diffère considérablement de la construction du roman.

\section{SOUS ET HORS LE JOUG DU PATRIARCAT}

Dans son roman, Roland Buti se plaît à représenter un cadre familial supposément caractéristique de la Suisse rurale de 1976 - soit cinq ans seulement après le résultat positif du vote au niveau fédéral en faveur du suffrage féminin - qui est initialement placé sous la stricte loi du patriarcat. La mère, silencieuse, effacée, est d'une fragilité qui
9 Ce mouvement de renforcement de la dureté du personnage de Gus, qui résulte peut-être du potentiel de jeu de l'acteur, s'observe au cours de l'écriture scénaristique et de la mise en scène sur le tournage: Gus se comporte en fin de compte davantage comme un adolescent rebelle que comme l'enfant qu'il était encore au stade du traitement.

10 Sur ce point, le romancier est pour le moins explicite:

"Maman a disparu. [...] Je n'ai pu détacher mon regard de la fenêtre noire, vide, comme orpheline sur la façade de la maison, imaginant avec une incompréhensible jalousie maman enroulée dans les draps à côté du corps puissant et calorique de papa" (/d., p.26). En fait, ainsi posée, cette jalousie est tout sauf "incompréhensible», du moins pour le lecteur; I'adjectif "orphelin» appliqué à l'objet qui canalise le fétichisme, la fenêtre, annonce le sentiment ultérieur de la perte de la mère. Cette situation voyeuriste est déplacée dans le film dans une séquence où Gus observe de loin sa mère juste avant qu'elle ne se couche avec son époux, alors qu'elle se peigne devant la glace. Le garçon qui aime non seulement lire des bandes dessinées comme dans le roman mais aussi dessiner (même si cette idée prégnante aux premiers stades du scénario est en fin de compte peu visible à I'écran) suit avec son doigt les contours de la silhouette maternelle comme s'il souhaitait se l'approprier, ce qui fait écho à un geste similaire montré au début du film, lorsque Gus, fasciné, déplace son doigt sur les photographies de corps féminins nus d'un magazine érotique qu'il a dérobé à l'épicerie (il s'agit du mensuel Emmanuelle, le magazine du plaisir, édité de septembre 1974 à août 1976) images en noir et blanc montrant des femmes qui s'enlacent, présageant ainsi la thématique de I'homosexualité féminine. 
11 À partir de la page 4 du traitement du 16 décembre 2014, le terme "colombe" apparaît à dix reprises dans le texte. Nos remerciements à Outside the Box ainsi qu'à Jeanne Rohner pour ses recherches menées dans les archives de production au cours de la préparation du contenu d'un atelier destiné à l'enseignement secondaire qui figure désormais dans l'offre du Centre d'études cinématographiques (CEC) proposée par l'intermédiaire du Service Culture et Médiation scientifique de I'UNIL.

12 À propos de la pertinence de I'étude du scénario dans une perspective d'étude sur le genre, voir notre ouvrage En cas de malheur, de Simenon à Autant-Lara (1956-1958): essai de génétique scénaristique. Récit, vedettes et représentations de genre, Genève, Droz, 2020.

13 R. Buti, op. cit., p. 30 . Une fois débutée sa liaison avec Cécile, ce rapport à l'espace se verra modifié, et ses difficultés respiratoires disparaîtront (/d., pp. 129-130); c'est au travers de telles touches que le romancier suggère une émancipation du personnage. /d., p. 31 .

Id., p. 68.

Id., pp. 76-77.

Id., p. 115.

Ibid.

Id., p. 148.

Id., p. 199

Nathalie Heinich, État de femmes. L'identité féminine dans la fiction occidentale, Paris, Gallimard, 1996. contraste avec un environnement "trop grand" pour elle, auquel elle ne semble pas pouvoir s'adapter: Buti nous dit d'elle qu'elle «évoluait égarée dans un monde de titans; tout ce qui était autour d'elle, la cuisinière, l'évier en pierre avec sa robinetterie très perfectionnée, les pots de grès alignés sur l'étagère, les poutres au plafond, la grande table de bois à laquelle j'étais assis, toute la pièce en somme avec ses murs massifs n'était pas à sa taille ${ }^{13}$. Dans ce portrait qui est prétexte à une description du lieu, c'est l'être même du personnage qui est identifié, fût-ce de manière contrastive, à l'espace domestique de la cuisine auquel est assignée l'épouse - si la mère s'affaire aux fourneaux, c'est à l'intention du fils assis à table. Certes, on nous dit qu'elle est "égarée ", puis que ces "tâches accaparantes [...] devaient l'empêcher de trop désespérer " ${ }^{14}$, ou encore, en faisant affleurer sa souffrance, qu'elle "est submergée par une vie intérieure intense ${ }^{15}$. Cependant, de cette intériorité-là, le lecteur ou la lectrice n'en savent rien, puisque le narrateur, explicitant les effets de la construction du point de vue choisi par l'auteur, confesse à propos de sa mère que, s'il peut décrire "sa vie minute par minute", il n'a "pas accès à ses pensées secrètes ${ }^{16}$. En fait, lectrice et lecteur sont invités à partager la même incompréhension à son égard que celle du personnage focal, qui vit la profonde déception de surprendre les ébats de sa mère et de Cécile, et de vivre brutalement un sentiment de perte qui, chez Gus, marque la sortie de l'enfance. Après que Rudy s'est brutalement jeté sur Cécile dont il a dans son esprit simple imaginé qu'elle partageait le désir qu'il ressent pour elle et que la mère de Gus est intervenue en utilisant le jet d'arrosage pour soustraire Cécile à l'emprise vigoureuse du jeune homme - complicité entre les deux femmes face à la violence masculine que Gus ne comprend pas en ne manifestant de l'empathie qu'à l'égard du jeune homme humilié ("Je l'ai regardé disparaître avec sa dégaine de paria qui n'a personne pour le consoler et j'ai détesté ces deux femmes") ${ }^{17}$, le fils doit faire face à "l'angoisse d'avoir perdu quelque chose pour de bon ${ }^{18}$. De son point de vue, elle s'est comportée en mère indigne; la complicité ou du moins l'empathie à l'égard de la figure paternelle s'est vue ainsi renforcée Gus assiste impuissant au spectacle d'un époux trahi dont il a pitié, tout en admirant le "moment de grâce virile ${ }^{19}$ lorsque le père, tel un cowboy de western, règle son compte par la force aux voisins qui se moquent très lourdement de la relation lesbienne de sa femme -, tandis que la mère est rejetée dans les limbes d'une étrangeté insondable, et se voit associée par Gus à la cause de tous les maux: à propos de son père consultant le baromètre, le narrateur précise qu'il «n'était pas le seul à considérer que le départ de maman était à l'origine de l'irruption du haut dans le bas, qu'il était la cause de cette soudaine perméabilité catastrophique", puis que lui-même, également, "pensai[t] que tout était très directement de sa faute $"^{20}$. Un passage-clé est celui où, après que la mère a annoncé à table avoir le projet d'aller travailler à la ville sur la suggestion de Cécile - «activité professionnelle porteuse d'identité " qui lui permettrait de sortir partiellement du statut de "femme liée" au sens de Nathalie Heinich ${ }^{21}$, la 
scénariste recourant dans son traitement au verbe (absent du roman) "s'émanciper» dans un discours indirect libre attribué à Léa («il est temps que sa mère s'émancipe, c'est une super idée!»22-, le père se jette sur l'amante de son épouse pour l'étrangler: la satisfaction ressentie par Gus est alors rendue avec une grande intensité ("Je jouissais de voir la panique dans les yeux de maman impuissante [...], je regardais le visage déformé de maman enlaidie par l'effroi et qui n'avait que ce qu'elle méritait »)23 face à cette démonstration d'une force virile qui reprend momentanément ses droits (la sœur tente vainement de venir en aide à Cécile). La scène de la tentative de strangulation est certes reprise dans le film, mais l'absence de représentation directe de l'intériorité de Gus (le film ne comporte aucune voix over) ne permet pas au spectateur ou à la spectatrice de saisir l'ampleur de la haine nourrie par Gus à l'égard de sa mère (de même que, dans la première partie, le caractère fusionnel de leur relation apparaît moins nettement); la focalisation interne du roman, même si elle n'exclut bien sûr pas une certaine distance en raison de l'âge du protagoniste - nous n'avons pas la naïveté de confondre sur un plan énonciatif le point de vue d'un personnage avec le discours véhiculé par l'œuvre romanesque, mais, ne jouant guère sur ce que les narratologues appellent un "narrateur non fiable", Le Milieu de I'horizon favorise I'assimilation de l'un à l'autre -, a selon nous pour incidence de renforcer la culpabilité de la mère. Le Gus du roman perpétue en outre sur Mado une violence que l'on pourrait dire transmise par le père (mais qui est présentée comme l'exutoire d'une agressivité résultant du départ de la mère), alors que le film l'atténue considérablement (et ce d'autant plus que Gus s'en excuse auprès de son amie, ce qui est radicalement différent du coup de sang représenté dans le livre ${ }^{24}$. Dans le chapitre final qui est consacré à une vente aux enchères du mobilier et du matériel du domaine après une ellipse de plusieurs années, le narrateur dit entrer «dans la maison avec l'espoir que papa s'y est retranché ", lui qui, "depuis qu'il vit seul, [...] a choisi de dormir dans la petite chambre vidée de tout le fatras qui l'encombrait ${ }^{25}$. Alors que le père y est décrit comme un être souffrant et que la dernière phrase du roman, poétique, se réfère aux sensations de cet homme "assis entre son fils et sa fille " dans ce qui fait de lui un individu profondément relié à sa terre (le roman lui attribue un discours sur son métier qui participe à la valorisation du personnage et à un rapport nostalgique aux valeurs conservatrices incarnées par ce dernier) ${ }^{26}$, aucun mot n'est dédié à la mère (pas même dans les dialogues entre Gus et sa sœur Léa), comme pour mieux signifier combien elle est sortie de leur vie. Une exception toutefois, indirecte: elle est mentionnée à travers Léa, dont le portrait de femme qui a réussi ne se départit pas, par ailleurs, d'une certaine ironie. Le narrateur devenu adulte fait le constat suivant: "Il y a dans son regard une parcelle d'âme qui ne lui appartient pas, une parcelle d'âme qu'elle a héritée de maman"; "c'est peutêtre pour cela que je suis gêné lorsqu'elle me fixe un peu trop longuement $"{ }^{27}$. La fin du film de Lehericey se situe sur ce point aux antipodes de la conclusion du roman, puisque non seulement il s'abstient I'horizon, traitement du 16 décembre 2014, p. 14.

23 R. Buti, op. cit., p. 133.

24 Id., pp. 182-185.

25 Id., p. 220.

26 Id., p. 234. La dernière phrase est la suivante: "Je me suis dit alors qu'il aurait voulu à cet instant précis être absorbé par le sol, aspiré avec douceur dans les profondeurs pour, enfin, s'amalgamer à toutes les reliques des hommes et des femmes nourris par ces terres autrefois fertiles ".

27 /d., pp. 224-225. 
Clémence Poésy a notamment joué dans des productions hollywoodiennes, par exemple aux côtés de Colin Farrell dans In Bruges (Bons baisers de Bruges, R.-U./E.-U., 2008) ou dans la série cinématographique des Harry Potter, ce qui lui confère une certaine notoriété auprès de la jeune génération susceptible de s'identifier à Gus.

29 Joanne Giger, Le Milieu de I'horizon, traitement du 16 décembre 2014, p. 3.

30 Joanne Giger, Le Milieu de I'horizon, continuité du 3 décembre 2015, p. 3. de s'appesantir sur les affres du père mais va jusqu'à mettre en scène une réconciliation de la mère et du fils. C'est sur cette séquence finale à propos de laquelle la réalisatrice dit dans l'entretien ci-dessous qu'elle a suscité beaucoup de débats dans ses discussions avec sa scénariste, et ce jusqu'au moment du tournage, que nous mettrons I'accent pour aborder la lecture particulière que les adaptatrices ont proposée du personnage de la mère.

\section{DE LA MAUVAISE MÈRE À LA FEMME ÉMANCIPÉE: LE FINALE DU FILM}

De manière générale, le film procède à une revalorisation des personnages de Cécile (incarnée elle aussi par une vedette) ${ }^{28}$ et de la mère de Gus, ne serait-ce qu'en raison de la plus grande proximité qui y est instaurée avec le couple. Elle se manifeste par exemple concrètement à l'image lorsque Gus et Mado surprennent les deux femmes qui s'étreignent (dans la voiture, et non nues au bord de la rivière) puisque, si la perception est initialement ancrée dans le regard de Gus situé à la lisière de la forêt, des plans rapprochés filmés dans le véhicule qui effleurent les corps en s'émancipant de l'ancrage dans le regard de l'enfant soulignent la complicité qui règne dans le couple.

Dans le traitement - c'est-à-dire dans un texte narratif d'un format restreint (ici 25 pages) comprenant peu de dialogues et dans lequel la voix narrative tend à expliciter les enjeux du récit en les condensant -, la position de la mère au sein de la famille au début de I'histoire est posée ainsi : "Discrète, Nicole semble parfois passer au second plan à la ferme: Léa ne la consulte jamais avant de changer le programme qu'elle écoute à la radio [...] et Jean, qui ne parle déjà pas beaucoup, se contente de l'embrasser furtivement avant sa journée de travail. II n'y a que Gus pour l'adorer en silence, mais le jeune garçon sent, depuis quelque temps, que quelque chose cloche ${ }^{29}$. Au vu de la valeur prescriptive d'un tel document - il ne s'agit pas d'une nouvelle littéraire, mais d'un texte qui programme un film à venir -, la modalisation du discours introduite par la formule "semble parfois" se réfère en fait à un état de choses qui est supposé devoir être perçu par le spectateur. Si cette intention est présente dans le film, elle n'y apparaît pas d'entrée de jeu. Dans la première continuité dialoguée d'une centaine de pages achevée par Joanne Giger une année après le traitement, le mal-être de la mère et sa marginalisation au sein de la famille sont considérablement atténués. Les premières indications relatives au portrait de Nicole figurant dans les didascalies sont toutefois différentes de celles qui seront suivies dans le film:

Des mains de femme posent un drap à côté d'un autre, puis disparaissent à nouveau. Enfin un visage apparaît. NICOLE (34), la mère de Gus, semble perdue dans ses pensées. Le petit garçon la contemple. Elle est jolie dans sa robe d'été, mais paraît ailleurs. Son visage s'éclaire lorsqu'elle aperçoit son fils. ${ }^{30}$ 
Gus, dans cette version du texte, offre des fleurs à sa mère, et aperçoit "un éclair de tristesse» dans les yeux de celle-ci. Dans le film, le fils sera représenté de manière moins enfantine, et sa mère, affairée à sortir du linge mouillé de la machine à laver avant de donner à boire aux lapins, ne sera pas montrée pensive ou attristée lorsque s'instaure avec Gus une interaction complice et ludique assortie de recommandations quant au fait qu'il doit être à la disposition de son père en cette période de tâches estivales. Les quelques dernières annotations qui vont dans le sens de l'expression de l'intériorité de la mère ("Nicole, pensive [...], lance un œil vers le ciel sans nuages ") ${ }^{31}$ dans une version ultérieure de la continuité établie quant à elle en collaboration avec la réalisatrice ne seront pas conservées en fin de séquence. L'accent est mis sur la joie ressentie par la mère, en dépit de la pénibilité des tâches, à s'amuser avec son fils. Ce sera d'ailleurs l'image de cette étreinte liminaire qui sera retenue pour l'affiche ${ }^{(\text {Fig.3) }}$, à partir d'une photographie de plateau permettant d'inclure les deux visages et de supprimer le contrejour, et qui a donc été jugée emblématique du discours du film. L'importance de cette relation maternelle se confirme jusqu'à la séquence finale, qui invalide l'assimilation opérée dans le roman entre le statut de "mauvaise mère" et l'émancipation féminine.

II s'agit bien d'un "finale" au sens musical du terme puisque les protagonistes se retrouvent au concert proposé par l'orchestre dont Léa fait partie et qui joue La Symphonie du Nouveau Monde de Dvorak, une musique très souvent utilisée au cinéma et dont la présence qui exacerbe les émotions est de la sorte motivée par une source diégétique - même si, de par son intensité et son caractère enveloppant, elle s'apparente à une musique extradiégétique (elle débute significativement déjà à la séquence précédente, le pont sonore ménageant une progression jusqu'au climax). Si le roman fait également mention de la même symphonie, il ne fait pas du concert son point d'orgue, ni même celui du récit de la jeunesse de Gus (qui s'achève sur l'enterrement de Rudy, où les victimes du drame sont uniquement associées à des personnages masculins). Ce qui s'y joue est exactement l'inverse du résultat auquel sont arrivées les adaptatrices: d'une part le rapprochement entre le père et le fils, qui tous les deux se sentent complètement étrangers aux autres personnes présentes («Tout le monde discutait de groupe en groupe un verre à la main, mais sans jamais pénétrer dans le cercle qui nous isolait de la foule assemblée " $)^{32}$, d'autre part la disparition complète de la mère de Gus dans sa fonction maternelle (alors même qu'elle est tout entière circonscrite dans le terme "maman ») qui est soulignée dans la négation de la dernière phrase de ce passage: "Maman ne s'est pas penchée sur moi ${ }^{33}$. De toute évidence, le récit de fiction atteste une forme d'exclusion réciproque entre la relation passionnelle (en l'occurrence homosexuelle, ce qui prend un sens particulier au vu des débats relatifs à l'homoparentalité, qui en Suisse sont contemporains de la parution du livre) et l'incapacité à assumer son rôle de mère: «Maman avait vingt et un an lorsque je suis né. J'avais maintenant tion avec Delphine Lehericey, Le Milieu de I'horizon, continuité du 18 mai 2018, p. 4.

32 R. Buti, op. cit., p. 206.

33 Id., p. 207. 


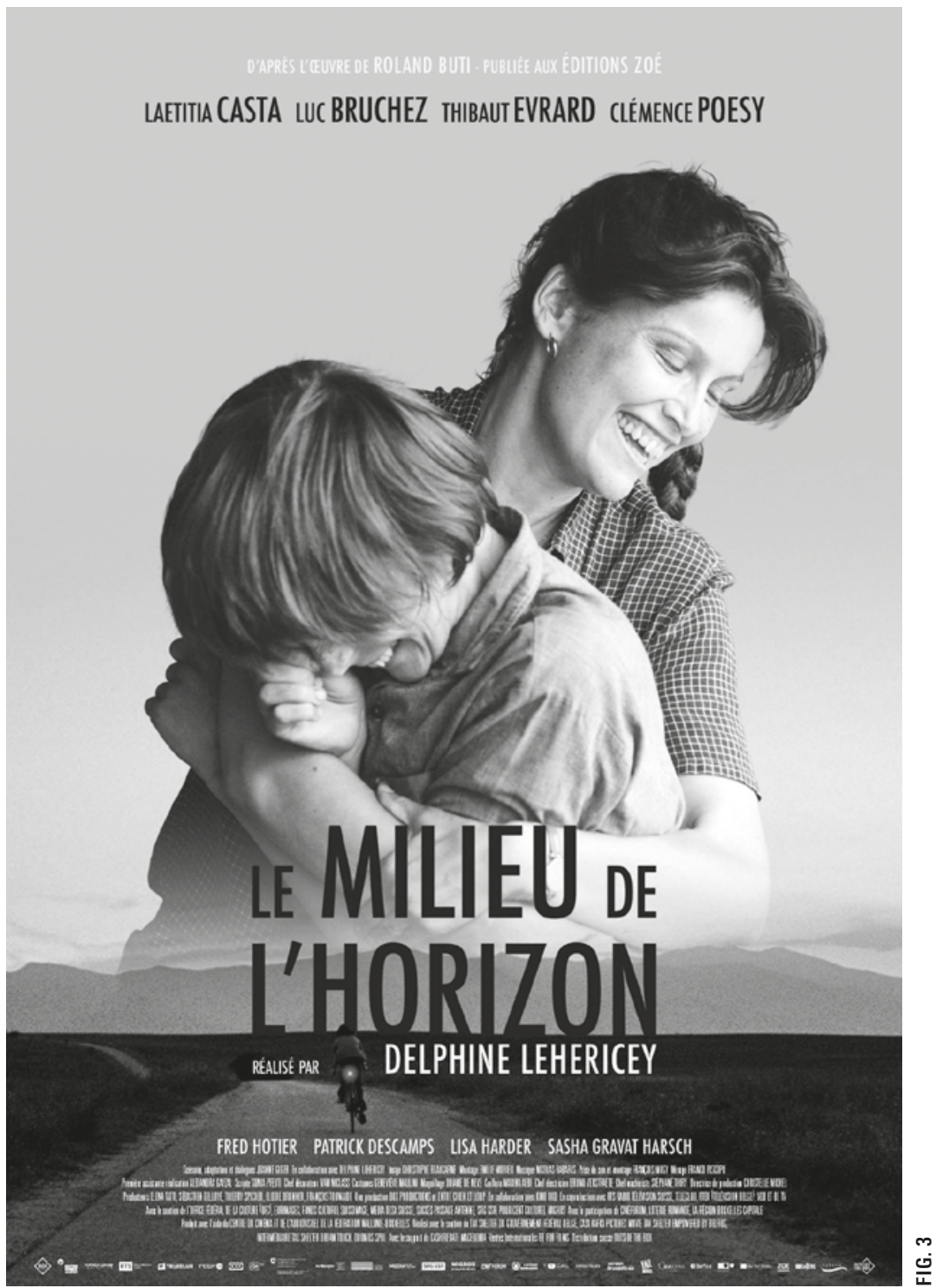

L'affiche du film: "l'horizon» qui se dessine est celui de la relation affectueuse entre la mère et son fils. 

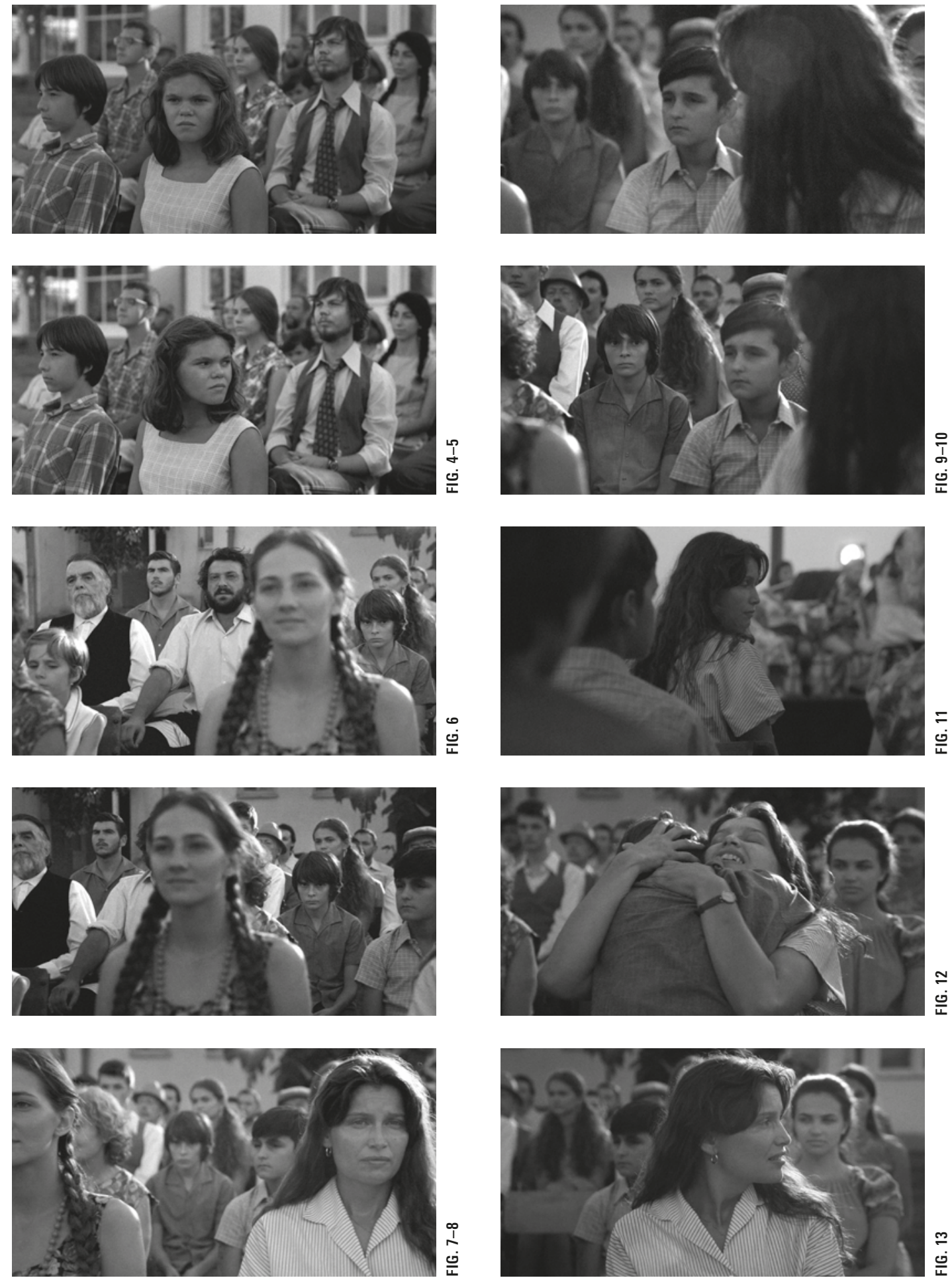
$34 \quad / d .$, p. 205

35 Pour ce type de remarques, nous nous référons aux rushes que Box Productions a accepté de mettre à notre disposition.

36 François Jost, L'CEil-caméra. Entre film et roman, Lyon, PUL, 1987. treize ans; elle nous abandonnait et elle avait peut-être à nouveau vingt et un ans comme si toute sa vie passée avec nous avait été une erreur, une parenthèse hors du temps ${ }^{34}$.

Dans le film, cette séquence conclusive, qui se présente également comme un écho à la première apparition de la mère (l'étreinte lorsque Gus revient de sa balade à vélo est rejouée dans ce finale) et comme une ouverture (Gus face à "l'horizon ", où l'âge adulte se présente comme "nouveau monde»), est caractéristique du soin apporté dans Le Milieu de I'horizon à la qualité de l'image (le choix de la production s'est porté sur de la pellicule $35 \mathrm{~mm}$, ce qui est rare en 2017) et du son, confiés l'un et l'autre à des techniciens renommés, respectivement le chef opérateur Christophe Beaucarne et le preneur de son François Musy. La séquence procède en effet d'une circulation entre les personnages présents parmi le public du concert au cours de laquelle se recrée le lien entre la mère et son fils. Le premier des relais est incarné par Mado qui, contrairement à ce qui est indiqué dans le scénario de tournage et même de ce qui a été tourné sans être conservé au montage ${ }^{35}$, ne rejoint pas Gus en le suivant dans sa course (ce qui l'amène à passer devant Cécile, qui ne se tient pas à proximité de Nicole mais l'attend dans le parking) en fin de séquence, se contentant d'amorcer la mise en relation des personnages: lorsqu'elle tourne la tête pour quitter des yeux les musiciens sur l'estrade et orienter son regard vers les chaises situées derrière

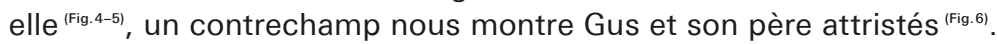
Un travelling latéral décentre ensuite cette composition pour faire sortir du cadre le père et y faire entrer la mère ${ }^{(\mathrm{Fig.7-8)}}$, assise au premier plan, tandis que la mise au point passe de l'arrière-plan à l'avant-plan (Gus devient flou), en un "mouvement " qui s'inverse ensuite lorsque

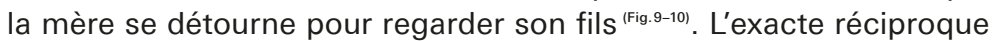
de ce plan avec la mère en amorce et l'enfant net dans l'image lui fait suite $^{(\text {Fig. 11): }}$ cette symétrie dit le lien indéfectible entre les deux êtres. Un autre plan comportant Gus en amorce se démarque par sa durée, et replace le segment filmique sous la domination de la focalisation interne sur Gus (en l'occurrence ici via une ocularisation interne secondaire puis primaire, pour reprendre la terminologie jostienne) ${ }^{36}$; le jeune homme prend alors dans le film - contrairement à ce qui était prévu dans les différents états du scénario, où il prenait immédiatement la fuite - la décision de rejoindre sa mère aux premiers rangs et


suite le lieu du concert, mais nous ne le suivons pas immédiatement: la caméra reste rivée sur la mère qui a le regard dirigé vers le horschamp droit du cadre, là où Gus est sorti du champ ${ }^{\text {(Fig. 13) }}$. Lorsqu'un sourire commence à se dessiner sur les lèvres de Nicole, signe de satisfaction devant la réconciliation qui vient d'advenir, la musique dans le film fait place à la musique $d u$ film, déjà entendue auparavant; ce plan qui réserve une place-clé aux émotions de la mère sur lesquelles se calquent celles transmises par le film - constitue donc, au seuil de l'épilogue, une forme de climax, et résulte d'une interprétation libre de l'œuvre originale qui va jusqu'à en prendre le 
contrepied. Ce changement, qui consacre une autre représentation de "maman» (devenue Nicole, une trentenaire en quête d'émancipation), est précédé d'une modification survenue sur le plateau et au montage: le scénario "de tournage" (version datée du 7 septembre $2018)^{37}$ prévoyait que Jean soit à l'origine de I'initiative de Gus de rejoindre sa mère - il le lui suggérait, les larmes aux yeux -, la réconciliation dépendant alors de l'adoubement du patriarche (et ce d'autant plus que, comme l'atteste une série de rushes, un travelling latéral sur la gauche mettait en évidence à ce moment-là I'homme à la barbe blanche assis à la droite de Jean, comme pour situer le propos dans une filiation). Avec la suppression de cette réplique du père, nous sommes encore plus loin du roman dans lequel Gus, en observant sa mère et Cécile (qui assiste au concert puis à la réception), ne peut se retenir de murmurer "Quelles salopes!" pour désigner les deux femmes associées à une déliquescence de la famille ${ }^{38}$, formule misogyne qui se trouve sous la plume de la scénariste dans son traitement puis disparaît dans les continuités dialoguées.

Les deux adaptatrices se sont emparées d'un roman écrit par un homme, et d'une histoire qui met en scène la perception par un garçon du vacillement d'un modèle traditionnel et patriarcal de la famille. Cette réappropriation du récit romanesque, qui a évolué au cours de la genèse du film, s'accompagne de parti-pris au niveau de la représentation des rapports sociaux de genre qui, sans faire du Milieu de I'horizon un film féministe, atténuent, comme nous avons pu l'observer sur la base d'une étude comparative, la prédominance des valeurs associées au masculin ainsi que la misogynie que le romancier, à force de les exposer par le biais de ses personnages, tend à poser ; le film, lui, les évince.
Précisons que le tournage a eu lieu du 20 juillet au 7 septembre (35 jours dont 27 jours en Macédoine et 8 en Belgique).

$38 \quad$ R. Buti, op. cit., p. 207. 